\title{
DEPOSITO DE DETRITO, BIOMASA Y PRODUCCION PRIMARIA NETA EN LOS BOSQUES DE LA LLANURA ALUVIAL INUNDABLE DE LA AMAZONIA PERUANA ${ }^{1}$
}

\section{Gustav Nebel ${ }^{2}$, Jens Gradsted ${ }^{2}$ y Angel Salazar Vega ${ }^{3}$}

\section{RESUMEN}

La densidad expresada en biomasa y el incremento de fustes y ramas en parcelas permanentes de muestreo, localizadas en tres bosques naturales maduros de la llanura aluvial inundable, adyacentes al río Ucayali, en la Amazonía Peruana, fueron estudiados durante un periodo de cuatro años. Adicionalmente, en las mismas parcelas de muestreo se recolectó, semanalmente, durante un año, el depósito caído de detrito fino. Los tres bosques, que localmente son conocidos como restinga alta, restinga baja y tahuampa, están situados en suelos aluviales ricos en nutrientes. La inundación en ellos promedió 1, 2 y 4 meses por año, respectivamente. El depósito de detrito fino fue alrededor de $700 \mathrm{~g} / \mathrm{m}^{2} /$ año en los tres bosques, de los cuales el $60 \%$ estuvo constituido por hojas. El depósito de detrito parece correlacionarse con la inundación y la precipitación, pero el factor más probable que induce la defoliación es la inhibición del crecimiento causado por la inundación. Las hojas que caen al final o justo después de la inundación, tuvieron áreas específicas foliares menores que aquellas hojas que caen durante el resto del año (aproximadamente $60 \mathrm{~cm}^{2} / \mathrm{g}$ versus $110 \mathrm{~cm}^{2} / \mathrm{g}$ ). El área foliar específica promedio anual se incrementó con la disminución promedio del período de inundación de los bosques. Asumiendo en 12 meses la

1 Traducción del original en inglés "Litter fall, biomass and net primary production in flood plain forests in the Peruvian Amazon".

2 Universidad Real de Veterinaria y Agricultura. Departamento de Economía y Recursos Naturales, Unidad de Forestales. Rolighedsvej 23, 1958 Frederiksberg C., Dinamarca. Teléf.: 45352822 32; Fax: 45352826 71. E-mail: gne@kvl.dk

3 Instituto de Investigaciones de la Amazonía Peruana. Avda. Abelardo Quiñónes km 2,5. Iquitos (Perú). 
duración de una hoja, el índice de área foliar se estimó en 4,2-4,4. Estos datos podrían incrementarse al efectuar las correcciones por pérdida a causa de herbívoros y por la clasificación errónea del detrito foliar como desperdicio. Para árboles con más de $10 \mathrm{~cm}$ DAP el volumen de madera de los tres tipos de bosque fue de $662-750 \mathrm{~m}^{3} / \mathrm{ha}$ y el incremento del volumen de madera fue de $23-28 \mathrm{~m}^{3} / \mathrm{ha} / \mathrm{año}$. Las densidades del volumen de madera pesada en los bosques fue de 0,55-0,66. El promedio de la biomasa viva sobre la superficie del suelo de los tres bosques fue de 34 493-48 $692 \mathrm{~g} / \mathrm{m}^{2}$. En la masa viva sobre el suelo se registró una producción primaria neta (PPN) de $2082-2558 \mathrm{~g} / \mathrm{m}^{2} / a n ̃ o$, sin contar las pérdidas por herbívoros. Este nivel concuerda con el rango observado en los bosques húmedos tropicales, pero es bajo en comparación a los bosques de la llanura aluvial inundable de la Amazonía brasileña, en donde se registró un PPN de hasta $3360 \mathrm{~g} / \mathrm{m}^{2} / \mathrm{año}$. Sin embargo, en los bosques de la llanura aluvial inundable brasileños se aplicó un método diferente de evaluación. En este estudio se registró un alto nivel de producción de biomasa en comparación con otros bosques lluviosos tropicales (1 709 versus $734 \mathrm{~g} / \mathrm{m}^{2} / \mathrm{año}$ ). Esto es significativo teniendo en cuenta que, durante las inundaciones, los procesos de crecimiento de los árboles son inhibidos por varios meses cada año y que este evento coincide con los meses de más alta precipitación.

Palabras clave: Zonas húmedas, área foliar específica, índice de área foliar, densidad de la madera, carbón.

\begin{abstract}
During a four-year period the biomass density and increment of stems and branches were studied in one-hectares permanent sample plots located in three ripe natural flood plain forests adjacent to the Ucayali river in the Peruvian Amazon. Additionally, on the same sample plots fine litter fall was collected on a weekly basis during one year. The three forests which are locally known as high restinga, low restinga and tahuampa are situated on nutrient rich alluvial soils. They were on the average inundated for 1, 2 and 4 months per year, respectively. The fine litter fall was around $700 \mathrm{~g} / \mathrm{m}^{2} /$ year in all three forests, by which around $60 \%$ was identified as leaves. The litter fall seemed to be correlated with both flooding and precipitation, but the most probable factor inducing leaf shedding was growth inhibition caused by flooding. Leaves falling at the end of and just after the inundation had lower specific leaf areas than leaves dropping during the rest of the year (approximately $60 \mathrm{~cm}^{2} / \mathrm{g}$ versus 110 $\mathrm{cm}^{2} / \mathrm{g}$ ). The average annual specific leaf area increased with decreasing average
\end{abstract}


flooding period of the forests. Under the assumption of a 12 month leaf duration the leaf area index was estimated to be 4,2-4,4. Corrections for herbivory losses and erroneous classification of leaf litter as trash would result in higher values. For trees larger than $10 \mathrm{~cm}$ diameter at breast height the wood volume of the three forest types was $662-750 \mathrm{~m}^{3} / \mathrm{ha}$, and the wood volume increment was $23-28 \mathrm{~m}^{3} / \mathrm{ha} / \mathrm{year}$. The volume weighted wood densities was $0,55-0,66 \mathrm{~g} / \mathrm{c} 3 \mathrm{~m}$ in the forests. The average aboveground living biomass of the three forests was $34493-48692 \mathrm{~g} / \mathrm{m}^{2}$. A NPP of $2082-2558 \mathrm{~g} / \mathrm{m}^{2} /$ year was registered in the aboveground living biomass, excluding losses to herbivory. This level was within the range observed in tropical moist forests, but low in comparison to flood plain forests in the Brazilian Amazon, where a NPP of up to $3360 \mathrm{~g} / \mathrm{m}^{2} /$ year was registered. However, in the Brazilian flood plain forests a differing method was applied. In the present study a high level of wood biomass production was registered in comparison to other tropical rain forests (1 709 versus $734 \mathrm{~g} / \mathrm{m}^{2} /$ year). This is noteworthy taking into consideration that growth processes of trees are inhibited several months each year during the flooding, and this event is coincident with the months of highest precipitation.

Key words: Wetlands, specific leaf area, leaf area index, wood density, carbon.

\section{INTRODUCCION}

Con el fin de entender como están organizados y como funcionan los ecosistemas forestales, es necesario conseguir información sobre su estructura y dinámica. Tal información es también un importante prerrequisito para el desarrollo de los sistemas de manejo adaptados. En esta responsabilidad es importante el rol de la densidad de biomasa y la producción primaria neta (PPN). Consecuentemente, desde años atrás, mucha de la investigación se ha concentrado en aspectos relacionados con estos parámetros asociados (por ej. Lieth y Whittaker, 1975; Murphy, 1975; UNESCO, 1978a, b; Medina y Klinge, 1983; Brown y Lugo, 1984; Jordan, 1983, 1985, 1989; Ryan et al., 1994; Brown, 1997; Base de Datos PPN, 1999). Sin embargo, aun existen problemas, especialmente los relacionados con la elección de la metodología y el muestreo en tiempo y espacio. Esto se hace más pronunciado en los complejos ecosistemas de bosque húmedo tropical, los cuales cubren una vasta área y por ende varían considerablemente en estructura, composición florística y dinámica de bosque (Whittaker y Marks, 1975; Proctor, 1983, 1984; Brown, 1997). Por ejemplo, debido a que las propiedades del subsuelo son difíciles de estudiar, muchos estudios se han concentrado en aspectos de la superficie del suelo (UNESCO, 1978a; Medina 
y Klinge, 1983; Base de Datos PPN, 1999). Las pérdidas de PPN por causa de herbívoros puede ser significativa, pero igualmente difícil de estimar (por ej. Coley, 1982; Janzen, 1983; Wint; 1983; Morrow, 1984).

Una mayor preocupación acerca de que los cambios climáticos son provocados por un elevado contenido de $\mathrm{CO}_{2}$ atmosférico, ha acentuado la necesidad de conocer la biomasa mundial y su dinámica. Se cree que las principales causas del mayor contenido de $\mathrm{CO}_{2}$ atmosférico, son las emisiones por combustión de fuentes fósiles de energía, pero otros factores, como los cambios en la cobertura de la vegetación, pueden también influir significativamente en el ciclo de carbono mundial (informes en Apps y Price, 1996). El rol de los bosques tropicales atrajo especial atención, sin embargo existen pocos datos que pueden ser usados en el desarrollo de modelos para el contenido y flujo del carbono (por ej. Brown y Lugo, 1984; informes en Apps y Prince, 1996; Brown, 1997).

Worbes (1997), estudió la densidad de biomasa y PPN de dos rodales jóvenes de bosque de la llanura aluvial inundable con suelos ricos en nutrientes cerca de Manaos, Brasil. Registró un promedio de densidades de biomasa moderado (25 800 y 27900 $\mathrm{g} / \mathrm{m}^{2}$ ) y altos niveles de PPN (2 380 y $3360 \mathrm{~g} / \mathrm{m}^{2} / \mathrm{año}$ ), sin contar con la pérdida por herbívoros. Sin embargo, la gran extensión de los bosques de la llanura aluvial inundable amazónica, justifica realizar posteriores estudios a fin de observar si se mantienen estos niveles en otros lugares y otros tipos de bosque. Este estudio contribuye con datos sobre densidad de biomasa, PPN y otros parámetros relacionados en tres tipos de bosque de la llanura aluvial inundable de la selva baja de la Amazonía Peruana. En las parcelas permanentes de muestreo, se monitoreó durante cuatro años la densidad de la biomasa viva en la superficie del suelo y el incremento de fustes y ramas. En las mismas parcelas de muestreo se recolectó semanalmente, durante un año, el depósito de detritos finos, lo cual permitió estimar el índice de área foliar (IAF).

\section{AREA DE ESTUDIO}

Los estudios de campo fueron realizados en tres bosques de la llanura aluvial inundable en las localidades de Braga-Supay y Lobillo, aproximadamente a $10 \mathrm{~km}$ al suroeste de Jenaro Herrera ( $\left.4^{\circ} 55^{\prime} \mathrm{S}, 73^{\circ} 44^{\prime} \mathrm{O}\right)$, en el Bajo Ucayali, en la Amazonía Peruana. En Braga-Supay se estudiaron los tipos de bosque de restinga alta y restinga baja, mientras que en Lobillo se estudió el tipo de bosque de tahuampa. En todos los casos la vegetación fue de bosque lluvioso primario alto y cerrado, aunque los árboles de 
las especies más valiosas pudieron haber sido talados hace más de 30 años. El bosque de restinga baja es probablemente una etapa de sucesión más joven que la de los demás bosques. Los tres bosques se inundan anualmente durante la creciente del río Ucayali que llega al máximo alrededor del mes de abril. El promedio aproximado de los periodos de inundación anual fue: restinga alta un mes, restinga baja dos meses y tahuampa cuatro meses. En el periodo 1966-1997 el promedio de precipitación anual fue de $2715 \mathrm{~mm} /$ año. Los meses menos lluviosos van de junio a setiembre con precipitaciones mensuales de 142-186mm. Los aspectos generales de los bosques de la llanura aluvial inundable peruana fueron descritos por Kvist y Nebel (1999), mientras que los detalles específicos de los lugares de estudio fueron dados por Nebel et al. (1999a, 1999b, 1999c).

\section{METODOS}

\subsection{Recolección de datos}

En 1993 se establecieron, en cada uno de los tres tipos de bosque de la llanura aluvial inundable, tres parcelas permanentes de muestreo de una hectárea. Cada parcela de muestreo estaba dividida en 25 subparcelas de 20x20m cada una, con excepción de una parcela en donde se establecieron 24 subparcelas. Se inventarió toda la vegetación con diámetro a la altura del pecho (DAP) por encima de los $10 \mathrm{~cm}$ (estrato superior; Nebel et al., 1999a) y en los dos bosques de restinga se elaboraron submuestras de la vegetación por encima de $1,5 \mathrm{~m}$ de altura y hasta $10 \mathrm{~cm}$ de DAP (sotobosque; Nebel et al., 1999b). En cada tipo de bosque se dejó una parcela permanente de muestreo como parcela de control sin tratamiento, en un experimento silvicultural. Los datos de la dinámica del bosque se colectaron durante el periodo 1993-1997 midiendo repetidamente todos los individuos en 1994, 1995 y 1997 (Nebel et al., 1999c). Los datos del inventario de 1993 se usaron para estimar la densidad de la biomasa viva sobre la superficie del suelo, mientras que la correspondiente PPN se estimó mediante mediciones repetidas en las parcelas sin tratamiento.

El estudio del depósito de detrito fino siguió básicamente las recomendaciones dadas por Proctor (1983). Dentro de cada una de las tres parcelas permanentes de muestreo no intervenidas, de los tres tipos de bosque, se colocaron, en 1997, 25 trampas de detrito en posiciones seleccionadas aleatoriamente en cada una de las 25 subparcelas de 20x20 m. En el caso en que la posición estuviera ocupada por un árbol, se colocó 
la trampa a un lugar cercano al seleccionado. Una canasta de $10 \mathrm{~cm}$ de fondo, elaborada con una malla metálica de $2 \mathrm{~mm}$ sujeta a una estructura de tubo de plástico, delimitaba una superficie de trampa de detrito de $50 \mathrm{~cm}^{2}$; luego, en la parte superior, se colocó una tela de tejido fino, a fin de retener adecuadamente el detrito fino que cayera. También, para cuando el agua de la inundación alcanzara las trampas, fueron colocados postes de $1 \mathrm{~m}$ de altura por encima del suelo, lo que permitió elevar las trampas por encima del agua y la vegetación circundante.

Semanalmente y en el periodo del 2/12/97 al 24/11/98, se colectó el detrito fino incluyendo pequeñas ramas con diámetros de hasta $2 \mathrm{~cm}$. Cada semana, se recolectaron las piezas de tela colocadas en la parte superior de la malla metálica de cada trampa, con su contenido, siendo reemplazadas por otras piezas de tela vacías. Para 25 de los 52 censos se dibujó en un papel los contornos de las hojas recolectadas con una proporción área/peso conocida. Estos fueron luego cortados y pesados. El detrito de cada trampa fue colocado entre hojas de periódico y puesto en una prensa que fue usada para secar el material de detrito en una secador de plantas a base de kerosene por espacio de 3-4 días. Las tres fracciones consistentes en hojas, partes reproductivas y pequeñas ramas, corteza y desperdicio fueron pesadas separadamente, inmediatamente después de secadas y almacenadas en bolsas de plástico. Una muestra representativa de detrito perteneciente a todo el periodo de recolección fue secada en estufa hasta alcanzar peso constante en un laboratorio y se determinó un factor de conversión para la relación entre el peso de la planta seca en prensa y peso seco en estufa. El área foliar específica (AFE) se determinó como la relación área de la hojalpeso de la hoja seca. Los datos fueron utilizados para estimar el depósito de detrito fino, AFE, IAF, biomasa, contenido de carbón y PPN en el estrato inferior del bosque (superficie del suelo) consistente en hojas, partes reproductivas y pequeñas ramas, corteza y desperdicios.

\subsection{Estimación de parámetros}

Para estimar el AFE, IAF, la biomasa y la PPN se aplicaron métodos no destructivos de muestreo, debido a que estos requirieron menos tiempo y se pudieron integrar mejor en un proyecto donde las parcelas de muestreo fueron usadas para otros propósitos (Kvist et al., 1999; Nebel et al., 1999a, 1999b, 1999c). Según Medina y Klinge (1983) se asumió una duración de vida de 12 meses para las tres fracciones de detrito fino de hojas, partes reproductivas y pequeñas ramas, corteza y desperdicios. Consecuentemente, tanto el estimado de IAF como de la biomasa, para este componente de bosque, son valores anuales promedio. 
Para cada una de las 52 recolecciones semanales se calcularon las contribuciones al IAF como el producto del AFE por el peso del detrito foliar. En las semanas donde el AFE no fue medido directamente, el estimado se hizo por extrapolación.

La biomasa viva por sobre la superficie del suelo, como la biomasa de las hojas, las partes reproductivas y pequeñas ramas, la corteza y los desperdicios, fueron estimadas como el depósito de detrito acumulado durante el año. La biomasa viva por sobre la superficie del suelo, como la de los fustes y ramas se calcularon en base a la suma de las biomasas de los árboles individuales. Se utilizó un factor de forma común de 0,6 para el volumen de los fustes y ramas de todas las especies (por ej. Muller y Nielsen, 1965; Cannell, 1984). La densidad de la madera de varias especies fue consultada en trabajos anteriores (Chichignoud et al., 1990; Kroll et al., 1994; Brown, 1997). Se estimó una relación DAP-altura para todas las especies representadas por más de 20 individuos, en las parcelas permanentes de muestreo. El resto de individuos fueron agrupados, determinándose una relación común. En todos los casos se utilizó la siguiente función logarítmica:

$\mathrm{h}=\mathrm{a}+\mathrm{b} \ln (\mathrm{d})$

en donde h es la altura y d es el DAP.

La PPN por sobre la superficie del suelo, excluyendo la pérdida por herbívoros, se calculó como la suma de la PPN de la fracción de detrito fino, del sotobosque y del bosque de estrato superior. La PPN de la fracción de detrito fino se encontró sumando las 52 recolecciones semanales de detrito fino. Los estimados de la PPN del sotobosque y del bosque de estrato superior se determinaron promediando dos biomasas periódicas (1993-1995 y 1995-1997). Cada incremento periódico fue calculado como la suma de los incrementos de los árboles individuales en las parcelas de muestreo.

El contenido de carbono de la biomasa se estimó como el 50\% de la biomasa seca en estufa (Brown, 1997).

\subsection{Análisis estadístico}

Las diferencias entre los tipos de bosque en relación a las sumas anuales de depósito de detrito fino calculado para cada subparcela fueron probadas en el SAS ${ }^{\circledR}$ procedimiento GLM, usando un análisis univariado de varianza con los tipos de bosque 
como factores fijos. Se ejecutaron comparaciones en pares utilizando la prueba de Tukey-Kramer. Pruebas similares se realizaron para el AFE y el IAF.

Los parámetros de depósito de detrito fueron sujetos a un análisis de varianza con el procedimiento GLM en un modelo mixto con el tipo de bosque, el tiempo y la combinación tipo de bosque $\mathrm{X}$ tiempo, considerados como factores fijos y las trampas por tipo de bosque como factores aleatorios.

A partir de las mediciones de 1993, se calcularon el volumen y la biomasa para cada subparcela de las nueve parcelas de una hectárea. Las diferencias entre los tipos de bosque fueron probadas con el procedimiento MIXTO usando un análisis de variancia univariado con el tipo de bosque como factor fijo y las parcelas de una hectárea localizadas dentro del tipo de bosque como efecto aleatorio.

Las diferencias entre los tipos de bosque en relación al incremento de volumen y a la biomasa fueron probadas en base a los promedios de dos incrementos periódicos (1993-1997 y 1995-1997) para cada subparcela, en las tres parcelas de una hectárea sin tratamiento. El procedimiento MIXTO fue utilizado con el tipo de bosque considerado como factor fijo y las subparcelas colocadas dentro del tipo de bosque como efecto aleatorio.

\section{RESULTADOS}

\subsection{Depósito de detrito fino y AFE}

El depósito total de detrito fino durante el periodo de estudio fue de $693-714 \mathrm{~g} / \mathrm{m}^{2} /$ año (Cuadro 1). La fracción foliar fue del $60 \%$ del total del depósito de detrito fino. El $16 \%$ estaba constituido por partes reproductivas y el $24 \%$ restante consistió de pequeñas ramas, corteza y desperdicios. No se observaron diferencias significativas entre los tipos de bosque, excepto para la fracción de pequeñas ramas, corteza y desperdicios, las cuales fueron significativamente más bajas en la tahuampa que en la restinga alta (Cuadro 1).

El depósito de detrito fino varió considerablemente durante todo el año y, al parecer, hubo una correlación entre la inundación del bosque y la precipitación. Cuando los bosques estuvieron inundados de enero a abril, el depósito de detrito fino se aceleró, decayendo después hasta julio. A partir de allí y durante todo el periodo seco del año, el depósito se estabilizó en un nivel más o menos constante (Figura 1). Se detectaron significativas interacciones relacionadas al depósito de detrito entre el tipo de bos- 
que y el tiempo, lo que implica que el patrón de tiempo del depósito de detrito en los bosques fue diferente.

El área foliar específica (AFE) promedio de las hojas, en la restinga alta, fue significativamente diferente del AFE de las hojas de tahuampa (Cuadro 2). Los valores de AFE durante los meses de marzo a junio fueron alrededor de $60 \mathrm{~cm}^{2} / \mathrm{g}$, mientras que los valores durante el resto del año fueron de aproximadamente $110 \mathrm{~cm}^{2} / \mathrm{g}$ (Figura 2). Se encontraron interacciones significativas entre el tipo de bosque y el tiempo con respecto al AFE de las colecciones de detrito foliar, lo que significa que el patrón del tiempo del AFE de las hojas depositadas fue diferente en los bosques. El índice de área foliar fue de 4,2-4,4 y no se encontraron diferencias significativas entre los tipos de bosque.

\subsection{Biomasa, carbono y PPN}

El volumen de las ramas y fustes del estrato superior vivo de los tres tipos de bosque varió de 662 a $750 \mathrm{~m}^{3} / \mathrm{ha}$ y el incremento de volumen fue de $23-28 \mathrm{~m}^{3} / \mathrm{ha} / \mathrm{año}$. Entre los tipos de bosque se encontraron estrechas diferencias significativas relacionadas con el volumen en pie $(\mathrm{P}=0,09)$. La densidad estimada del volumen de madera pesada para el inventario de 1993 fue de 0,55 en la restinga alta, pero alcanzó valores considerablemente altos de 0,62 y 0,66 en la restinga baja y la tahuampa, respectivamente (Cuadro 3).

Cuadro 1. Depósito anual de fracciones de detrito fino recolectados en 25 trampas de 52 colecciones semanales en cada uno de los tres tipos de bosque durante el periodo 2/12/97-24/11/98. Promedio y límites al 95\% de intervalo de confianza.

\begin{tabular}{lcccc}
\hline & $\begin{array}{c}\text { Restinga alta } \\
\left(\mathbf{g} / \mathbf{m}^{2} / \mathbf{a n ̃ o}\right)\end{array}$ & $\begin{array}{c}\text { Restinga baja } \\
\left(\mathbf{g} / \mathbf{m}^{2} / \mathbf{a n ̃ o}\right)\end{array}$ & $\begin{array}{c}\text { Tahuampa } \\
\left(\mathbf{g} / \mathbf{m}^{2} / \mathbf{a n ̃} \mathbf{n}\right)\end{array}$ & $\begin{array}{c}\text { Total } \\
\left(\mathbf{g} / \mathbf{m}^{2} / \mathbf{a n ̃ o}\right)\end{array}$ \\
\hline $\begin{array}{l}417 \pm 20 \mathrm{a} \\
\text { Hojas }\end{array}$ & $430 \pm 22 \mathrm{a}$ & $411 \pm 22 \mathrm{a}$ & $419 \pm 12$ \\
$\begin{array}{l}\text { Pequeñas ramas, corteza y } \\
\text { desperdicios }\end{array}$ & $187 \pm 15 \mathrm{a}$ & $169 \pm 11 \mathrm{ab}$ & $159 \pm 9 \mathrm{~b}$ & $172 \pm 7$ \\
$\begin{array}{l}\text { Partes reproductivas } \\
\text { Total }\end{array}$ & $97 \pm 13 \mathrm{a}$ & $115 \pm 12 \mathrm{a}$ & $123 \pm 16 \mathrm{a}$ & $112 \pm 8$ \\
\cline { 2 - 5 } & $702 \pm 37 \mathrm{a}$ & $714 \pm 35 \mathrm{a}$ & $693 \pm 25 \mathrm{a}$ & $703 \pm 18$ \\
\hline
\end{tabular}

Los números con las mismas letras no son diferentes significativamente para un $\propto=0,05$. 
Cuadro 2. Promedio anual de área foliar específica de las hojas recolectadas por 25 trampas en 25 recolecciones semanales de tres tipos de bosque de la llanura inundable durante el periodo 2/12/97 - 24/11/98. Indice de área foliar de los mismos bosques de llanura inundable. Se asume una duración de la hoja de 12 meses. El índice de área foliar se calculó como el producto del área foliar específica por el peso del detrito foliar de cada una de las 52 recolecciones semanales. Promedios y limites de confianza al $95 \%$.

\begin{tabular}{lcccc}
\hline & Restinga alta & Restinga baja & Tahuampa & Total \\
\hline $\begin{array}{l}\text { Área foliar específica } \\
\left(\mathrm{cm}^{2} / \mathrm{g}\right)\end{array}$ & $102,9 \pm 3,7 \mathrm{a}$ & $98,2 \pm 3,5 \mathrm{ab}$ & $94,7 \pm 3,8 \mathrm{~b}$ & $98,6 \pm 2,1$ \\
Índice de área foliar & $4,4 \pm 0,2 \mathrm{a}$ & $4,4 \pm 0,3 \mathrm{a}$ & $4,2 \pm 0,2 \mathrm{a}$ & $4,3 \pm 0,1$ \\
\hline
\end{tabular}

Los números con las mismas letras no son diferentes significativamente para un $\propto=0,05$.

La biomasa de los fustes y ramas vivas sobre la superficie del suelo de los estratos superiores de bosque fueron de 34493 a $48691 \mathrm{~g} / \mathrm{m}^{2}$ (Cuadro 4) y los valores para el bosque de tahuampa fueron significativamente más altos que para los bosques de restinga. El promedio total de la biomasa viva por sobre la superficie del suelo fue de $44034 \mathrm{~g} / \mathrm{m}^{2}$, correspondiéndole $22017 \mathrm{~g} / \mathrm{m}^{2}$ al contenido de carbono (Cuadro 4). En promedio, la biomasa del sotobosque y de la fracción de detrito fino constituyeron el $8,3 \%$ y el $1,6 \%$ del total de la biomasa, respectivamente. 
Cuadro 3. Volumen en pie e incremento del volumen en pie de los fustes y ramas del estrato superior vivo sobre la superficie del suelo en los tres tipos de bosques de la llanura aluvial inundable. Densidad del volumen de madera pesada de los tres tipos de bosques de la llanura aluvial inundable calculados a partir de las densidades de la madera dados por Chichignoud et al. (1990), Kroll et al. (1994) y Brown (1997). Promedio y límites de confianza al $95 \%$.

\begin{tabular}{lccc}
\hline & $\begin{array}{c}\text { Restinga } \\
\text { alta }\end{array}$ & $\begin{array}{c}\text { Restinga } \\
\text { baja }\end{array}$ & Tahuampa \\
\hline $\begin{array}{l}\text { Volumen de fuste y ramas del estrato } \\
\text { superior vivo }\left(\mathrm{m}^{3} / \mathrm{ha}\right)\end{array}$ & $654 \pm 86 \mathrm{a}$ & $618 \pm 97 \mathrm{~b}$ & $773 \pm 68 \mathrm{a}$ \\
$\begin{array}{l}\text { Incremento en volumen de fuste y ramas del } \\
\text { estrato superior vivo }\left(\mathrm{m}^{3} / \mathrm{ha} / \mathrm{anno}\right)\end{array}$ & $23 \pm 4 \mathrm{a}$ & $28 \pm 5 \mathrm{a}$ & $26 \pm 2 \mathrm{a}$ \\
$\begin{array}{l}\text { Densidad del volumen de madera pesada } \\
\left(\mathrm{g} / \mathrm{cm}^{3}\right)\end{array}$ & 0,55 & 0,62 & 0,66 \\
\hline
\end{tabular}

Los números con letras iguales no difieren significativamente para un $\propto=0,05$.

La PPN de la biomasa viva de los fustes y ramas en los estratos superiores del bosque fue de $1185-1675 \mathrm{~g} / \mathrm{m}^{2} / a n ̃ o$. En los sotobosques de la restinga la PPN fue de 191$254 \mathrm{~g} / \mathrm{m}^{2} /$ año para toda la biomasa de fustes y ramas vivas por sobre la superficie del suelo. Entre los tipos de bosque se detectaron, a partir del PPN, estrechas diferencias significativas en los estratos superiores $(\mathrm{P}=0,07)$. 
Cuadro 4. Biomasa viva por sobre la superficie del suelo de los diferentes estratos del bosque en los tres tipos de bosque de la llanura aluvial inundable. Los valores para las fracciones de fuste y rama del estrato superior y del sotobosque se basaron en cuatro inventarios forestales realizados durante 1993-1997 en parcelas permanentes de muestreo de una hectárea. Los valores para la fracción de detrito fino fueron estimados a partir del depósito anual de detrito fino recolectado por 25 trampas en 25 recolecciones semanales en los tres tipos de bosque durante el periodo 2/12/97 24/11/98. El contenido de carbón fue estimado como el 50\% de la biomasa: Seca a la estufa. Promedios y límites de confianza al $95 \%$.

\begin{tabular}{|c|c|c|c|c|}
\hline & $\begin{array}{c}\text { Restinga alta } \\
\left(\mathrm{g} / \mathbf{m}^{2} /\right)\end{array}$ & $\begin{array}{c}\text { Restinga baja } \\
\left(\mathrm{g} / \mathbf{m}^{2} /\right)\end{array}$ & $\begin{array}{c}\text { Tahuampa } \\
\left(\mathrm{g} / \mathbf{m}^{2} /\right)\end{array}$ & $\begin{array}{l}\text { Total } \\
\left(\mathrm{g} / \mathbf{m}^{2} /\right)\end{array}$ \\
\hline $\begin{array}{l}\text { Biomasa de fuste y } \\
\text { ramas del estrato } \\
\text { superior vivo }\end{array}$ & $34493 \pm 3740 a$ & $35793 \pm 6311 a$ & $48691 \pm 4312 b$ & $39682 \pm 2894$ \\
\hline $\begin{array}{l}\text { Biomasa de fuste } \\
\text { y ramas del } \\
\text { sotobosque }\end{array}$ & $3686 \pm 421 a$ & $3610 \pm 386 a$ & - & $3649 \pm 269$ \\
\hline $\begin{array}{l}\text { Biomasa viva de la } \\
\text { fracción de detrito } \\
\text { fino }\end{array}$ & $702 \pm 37 \mathrm{a}$ & $714 \pm 35 a$ & $693 \pm 25 a$ & $703 \pm 18$ \\
\hline $\begin{array}{l}\text { Biomasa viva por } \\
\text { sobre la superficie } \\
\text { del suelo }\end{array}$ & 38881 & 40117 & $49384^{1}$ & 44034 \\
\hline $\begin{array}{l}\text { Contenido de } \\
\text { carbono de la } \\
\text { biomasa viva por } \\
\text { sobre la superficie } \\
\text { del suelo }\end{array}$ & 19441 & 20059 & $24692^{1}$ & 22017 \\
\hline
\end{tabular}

1 No se incluye la biomasa viva del sotobosque.

Los números con las mismas letras no son diferentes significativamente para un $\propto=0,05$ 


\section{DISCUSION}

\subsection{Depósito de detrito fino}

Se sabe que la inundación de los árboles en los bosques de la llanura aluvial inundable causa la latencia cambial (Gill, 1970; Crawford, 1982; Hook, 1984; Kozlowski, 1984; Junk, 1989; Schluter et al., 1994; Armstrong et al., 1994; Worbes, 1997). Asimismo, el crecimiento y desarrollo de las hojas pueden ser inhibidos durante la inundación y a menudo sucede una extensiva abscisión de las hojas (Kozlowski, 1984; Richards, 1996). De acuerdo a estas observaciones, en el presente estudio se observó, durante la inundación, una mayor caída de las hojas (Figura 1), similares resultados se obtuvieron en bosques de la llanura aluvial inundable de Brasil (Worbes, 1986, 1997). Las interacciones significativas entre tipos de bosque y tiempo, con respecto al depósito de detrito, pueden haber sido causadas por la ocurrencia de la inundación en diferentes épocas en los tres tipos de bosque, lo que redunda en diferentes épocas de mayor depósito de detrito. También se ha reportado correlación entre la caída de hojas y los periodos relativamente secos (por ej. Wright y Cornejo, 1990; Richards, 1996), aunque en un experimento donde se regaron los bosques durante el periodo seco, no se pudo demostrar que la sequía favoreciera la caída de las hojas (Wright y Cornejo, 1990). En el presente estudio, la mayor caída de hojas coincidió con la inundación y con los meses más lluviosos del año (Figura 1; Kvist y Nebel, 1999).

Comparado con otros estudios, el depósito de detrito fino en las localidades de Braga-Supay y Lobillo fue modesto. Los $700 \mathrm{~g} / \mathrm{m}^{2} / \mathrm{año}$ contrastan con los valores que a menudo exceden los $900 \mathrm{~g} / \mathrm{m}^{2} /$ año de otros bosques lluviosos de la selva baja tropical (por ej. UNESCO, 1978a; Leigh y Windsor, 1982; Jordan, 1983, 1985, 1989; Medina y Klinge, 1983; Proctor, 1984) y los $1060 \mathrm{~g} / \mathrm{m}^{2} / \mathrm{año}$ de un bosque comparativo de la llanura aluvial inundable amazónica de Manaos (Worbes, 1997). Los estudios de depósitos de detrito, generalmente, se han basado en muestras demasiado pequeñas que solamente representaban una porción de año (Proctor, 1983). En este estudio se aplicó un número relativamente grande de trampas, así como se hicieron recolecciones frecuentes en todo el periodo de un año con el fin de obtener una muestra satisfactoria de recolecciones de detrito que fuera más representativa tanto en tiempo como en espacio. De esta manera, se obtuvieron estimados estadísticamente aceptables de depósito de detrito (Cuadro 1) y se detectó una considerable variación anual (Figura 1). Bajo las condiciones de campo puede ser difícil conseguir recolectar depósito de detrito fino seco al horno a peso constante. Sin embargo, tal como lo 
señaló Proctor (1983), pueda que se tenga grandes diferencias en los estimados de depósito de detrito ocasionados por las diferencias en los métodos de secado aplicados. En el presente estudio se registró una pérdida de peso promedio de 8,6\% en las recolecciones de detrito que fueron secadas a peso constante en el laboratorio luego de haberlos dejado secar por varios días en las prensas. Por lo que, a fin de corregir el peso de las recolecciones de depósito de detrito, se desarrolló una función.

Solamente alrededor del $60 \%$ del total de depósito de detrito fino fue registrado como detrito foliar, el cual tuvo una baja proporción si se le compara con los resultados de otros estudios (por ej. Medina y Klinge, 1983; Proctor, 1983, 1984). En este estudio, sin embargo, algunas pequeñas piezas de hojas fueron clasificadas como desperdicio, debido a que se consideró que no todo el material fácilmente identificable pertenecía a esta fracción. Por lo tanto, se debe tener cuidado al comparar estos resultados con los de otros estudios en los cuales no se aplicó la fracción de desperdicio (Proctor, 1983).

La media anual de AFE de alrededor de $100 \mathrm{~cm}^{2} / \mathrm{g}$ fue comparable a los resultados reportados para otros bosques lluviosos de selva baja tropical, aunque fue más alta que la observada en lugares de bosque lluvioso de selva baja con suelos pobres en nutrientes (Medina y Klinge, 1983; Jordan, 1985, 1989). La variación durante el año muestra que las hojas más pesadas con bajos valores de AFE caen de los árboles durante y justo después de la inundación de los bosques (Figura 1 y 2). Debido a que tales hojas están usualmente asociadas con especies siempre verdes o con lugares de dosel expuesto al sol (Medina y Klinge, 1983) y que pueden ser adaptaciones a medio ambientes rigurosos (Grubb, 1977; Jordan, 1989), el depósito de tales hojas durante la inundación sugiere que la inundación causa tensiones altas. Además, los valores de AFE, que observados para las hojas de los bosques con la mayor inundación son significativamente más bajos, apoyan esta hipótesis (Cuadro 2).

El IAF puede variar considerablemente en tiempo y espacio, especialmente en los bosques lluviosos tropicales florística y estructuralmente heterogéneos (Medina y Klinge, 1983). En este estudio no se observaron diferencias significativas en IAF entre los tipos de bosque. Comparado con otros estudios de bosques húmedos tropicales donde se consideran normales los valores de IAF de 5-10 (Whittaker y Likens, 1975; Medina y Klinge, 1983; Jordan, 1985, 1989; Base de Datos PPN, 1999), los valores de 4,2-4,4 del presente estudio fueron bajos. Sin embargo, esta desviación puede ser en parte explicada por factores relacionados a los métodos aplicados. En otros estudios el IAF fue medido directamente con métodos destructivos o fue calculado en base a las recolecciones de detrito foliar que cubrían una porción del año 
solamente (Medina y Klinge, 1983). Tal como se explicó líneas arriba, la aplicación de la fracción de desperdicio en este estudio puede haber excluido de los datos de cálculo de IAF una parte del depósito de las hojas. Además, las pérdidas por herbívoros de porciones de hojas, pueden ser considerables (por ej. Coley, 1982; Janzen, 1983; Wint, 1983; Morrow; 1984), pero en esta investigación no se hicieron las correcciones pertinentes. Por otro lado, asumir la duración de vida de 12 meses de las hojas es de capital importancia al estimar el IAF en este estudio y las desviaciones pueden haber influenciado significativamente en los resultados.

\subsection{Biomasa y PPN}

Los valores totales de biomasa viva por sobre la superficie del suelo del presente estudio estuvieron dentro del rango común, comparado con los de otros bosques lluviosos de selva baja tropical -pero en sus límites superiores- y, especialmente, en comparación con los bosques de lugares de suelos pobres en nutrientes (Whittaker y Likens, 1975; Medina y Klinge, 1983; Brown y Lugo, 1984; Jordan, 1985,1989; Brown, 1997; Base de Datos de PPN, 1999). Las diferencias significativas entre tipos de bosque con respecto a sus densidades de biomasa, que muestran densidades crecientes con la llegada del periodo de inundación, son causadas principalmente por las diferencias entre las densidades de la madera de los bosques, aunque también, sin embargo, se encontraron grandes diferencias en el volumen de madera (Cuadro 4). El volumen de madera relativamente bajo del bosque de restinga baja se podría deber a su etapa de sucesión joven, lo cual está de acuerdo con las indicaciones florísticas observadas por Nebel et al. (1999a). Sin embargo, las diferencias en densidades de la madera entre los tipos de bosque son difíciles de explicar y en parte contradicen las observaciones hechas por Worbes (1997), quién encontró densidades de madera crecientes en relación a la etapa de sucesión.

La PPN por sobre la superficie del suelo en los bosques de Braga-Supay y Lobillo, excluyendo las pérdidas por herbívoros, fue de $2082-2558 \mathrm{~g} / \mathrm{m}^{2} / \mathrm{año}$. Estos datos se encuentran en el rango de valores reportados para otros bosques lluviosos de selva baja tropical -en el límite superior- (por ej. Murphy, 1975; Whittaker y Likens, 1975; UNESCO, 1978a; Medina y Klinge, 1983; Jordan, 1983, 1985, 1989; Base de Datos de PPN, 1999). Por otra parte, esto difirió con el valor para un rodal de bosque semejante de 80 años de la llanura aluvial inundable brasileña estudiado por Worbes (1997), éste registró una PPN de $3360 \mathrm{~g} / \mathrm{m}^{2} / \mathrm{año}$, excluyendo las pérdidas por herbívoros. Sin embargo, esta diferencia puede deberse, en parte, a los cálculos de PPN efectuados por Worbes (1997). Para obtener un estimado de la PPN él sumó los 
valores para el depósito de detrito fino, depósito de madera muerta y el incremento de madera registrado en diferentes estudios de bosques de la llanura aluvial inundable. Comparándolo con los métodos aplicados en este estudio, ello resultó ser una sobre estimación de la PPN, debido a que deben haber sido contados ya sea el depósito de madera muerta (asumiendo que el total de biomasa se encuentra en situación estable) o el incremento de madera, a fin de proveer resultados semejantes, no ambos. Al utilizar solamente uno de estos dos datos, la PPN para el mencionado rodal de zona inundable fue de $2220-2500 \mathrm{~g} / \mathrm{m}^{2} / \mathrm{año}$, lo cual concuerda con los resultados de este estudio.

Muller y Nielsen (1965) compararon la productividad en un bosque húmedo tropical con la de un bosque templado de abedul. Ellos encontraron niveles semejantes de producción de biomasa de madera en los dos bosques $\left(900\right.$ y $960 \mathrm{~g} / \mathrm{m}^{2} / \mathrm{año}$, respectivamente), a pesar de tener una producción primaria mucho más alta en el bosque húmedo tropical. Jordan (1983) revisó los estudios de PPN de los bosques húmedos tropicales, también estudió la relación entre la madera y la producción de detrito en ecosistemas forestales que se presentan bajo diferentes balances de radiación de la superficie de la tierra. Encontró que las proporciones de producción de detrito fueron las más bajas en las regiones con los más bajos balances de radiación y que las proporciones aumentaron con el aumento del balance de radiación. Sin embargo, no se pudieron detectar diferencias significativas entre la producción de madera de diferentes regiones. Por ejemplo, en los estudios mencionados por Jordan (1983), el promedio de producción de madera y de detrito en lugares con el más alto balance de radiación en la superficie de la tierra, fue de 743 y $975 \mathrm{~g} / \mathrm{m}^{2} / a n ̃ o$, respectivamente. La conclusión fue que la más alta PPN pertenece a las regiones tropicales, pero que la producción de madera estaba al mismo nivel que en las regiones templadas.

Los hallazgos de este estudio contrastan con estos resultados. En los bosques de

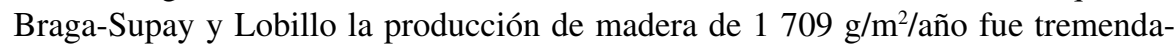
mente más alta y la producción de detrito de $703 \mathrm{~g} / \mathrm{m}^{2} /$ año fue más baja, dando como resultado una diferente relación de producción madera/detrito. Teniendo como base estos datos, no se puede saber si esto se debe a que los bosques se encuentran en una etapa de sucesión caracterizada por una alta producción de madera o a las características inherentes de los bosques de la llanura aluvial inundable que crecen en suelos aluviales ricos en nutrientes. Debido a que los tres tipos de bosque habían entrado a una fase de dinámica de espera y tenían elementos florísticos que pertenecen a las fases secundarias (Nebel et al., 1999a), parecía más probable que la productividad de madera fuera simplemente alta. Es relevante indicar que este nivel fue alcanzado en un medio ambiente donde los procesos de crecimiento fueron obstaculizados durante 
una gran parte del año, que además coincidió con el periodo de alta precipitación. Sin embargo, la producción de biomasa leñosa encontrada en base a los incrementos radiales, en un bosque brasileño de llanura aluvial inundable semejante, fue más baja (720 g/m²/año; Worbes, 1997), lo cual enfatiza la necesidad de desarrollar más estudios sobre la productividad en los bosques de la llanura aluvial inundable amazónica.

\section{RECONOCIMIENTOS}

Al Instituto de Investigaciones de la Amazonía Peruana (IIAP) y su estación de campo del Centro de Investigaciones Jenaro Herrera (CIJH) quienes amablemente proporcionaron las facilidades y la logística de campo. Estamos en deuda con el personal del CIJH y los pobladores de Jenaro Herrera por aceptar y contribuir a la culminación del trabajo de campo, especialmente a Arístides Vásquez y Julio Irarica quien pacientemente recolectaron y secaron las muestras de detrito fino. Anders Ræbild realizó un comentario en una versión preliminar del manuscrito. El y Brigitte Ron proporcionaron consejos en los análisis estadísticos. Los fondos fueron proporcionados por la Agencia Danesa de Desarrollo Internacional (DANIDA).

\section{BIBLIOGRAFÍA}

APPS, M.J.; PRICE, D.T. 1996. Forest ecosystems, forest management and the global carbon cycle. Berlin: Springer. 452 pp.

ARMSTRONG, W.; BRÁNDLE, R.; JACKSON, M.B. 1994. Mechanisms of flood tolerance in plants. In: Acta Botanica Neerlandica, 43(4): 307-358.

BROWN, S. 1997. Estimating biomass and biomass change of tropical forests. Rome: FAO Forestry Paper, 134. 55 pp.

BROWN, S.; LUGO, A.E. 1984. Biomass of tropical forests: A new estimate based on forest volumes. In: Science, 223: 1290-1293.

CANNELL, M.G.R. 1984. Woody biomass of forest stands. In: Forest Ecology and Management, 8:299-312. 
CHICHIGNOUD, M.; DÉON, G.; DÉTEINNE, P.; PARANT, B.; VANTONUNE, P. 1990. Tropical timber atlas of Latin America. Yokohama: ITTO/CTFT. $218 \mathrm{pp}$.

COLEY, P.D. 1982. Rates of herbivory on different tropical trees. In: E.G. Leigh; S. Rand; D.M. Windsor (editors). The ecology of a tropical forest. Seasonal rhythms and long-term changes. Washington: Smithsonian Institution Press. pp. 123-132.

CRAWFORD, R.M.M. 1982. Physiological responsos to flooding. In: Enc. Plant Physiol, 12B: 453-477.

GILL, C.J. 1970. The flooding tolerance of woody species - a review. In: Forestry Abstracts, 31(4): 671-688.

HOOK, D.D. 1984. Adaptations to flooding with fresh water. In: T.T. Kozlowski (editor). Flooding and plant growth. Academic Press. pp. 265-294.

JANZEN, D.H. 1983. Food webs: who eats what, why, how, and with what effects in a tropical forest? In: F.B. Golley (editor). Tropical rain forest ecosystems. Structure and function. Amsterdam: Elsevier Scientific Publishing Company. pp. 167-182.

JORDAN, C.F. 1983. Productivity of tropical rain forest ecosystems and the implications for their use as future wood and energy sources. In: F.B. Golley (editor). Tropical rain forest ecosystems. Structure and function. Amsterdam: Elsevier Scientific Publishing Company. pp. 117-136.

1985. Nutrient cycling in tropical forest ecosystems. Wiley, Chichester.

1989. An Amazonian rain forest. The structure and function of a nutrient stressed ecosystem and the impact of slash-and-burn agricultura. UNESCO. $176 \mathrm{pp}$.

JUNK, W.J. 1989. Flood tolerance and tree distribution in central Amazonian floodplains. In: L.B. Holm-Nieisen; I.C. Nielsen; H. Baislev (editors). Tropical forests. Botanical dynamics, speciation and diversity. Academic Press Limited. pp. 47-64. 
KVIST, L.P.; NEBEL, G. 1999. A review of Peruvian flood plain forests: Ecosystems, inhabitants and resource use. Copenhagen: Royal Veterinary and Agricultural University, Department of Economics and Natural Resources, Unit of Forestry. Unpublished manuscript.

KVIST, L.P.; ANDERSEN, M.K.; STAGEGAARD, J.; HESSELSOE, M.; LLAPAPASCA, C. 1999. Extraction from woody forest plants in flood plain communities in Amazonian Peru: Evaluation, choice and conservation status of resources. Copenhagen: Royal Veterinary and Agricultural University, Department of Economics and Natural Resources, Unit of Forestry. Unpublished manuscript.

KOZLOWSKI, T.T. 1984. Responses of woody plants to flooding. In: T.T. Kozlowski (editor). Flooding and plant growth. Academic Press. pp. 129-163.

KROLL, B.S.; NALVARTE, W.A.; MARMILLOD, D. 1994. Arboles del Perú. Especies forestales de Dantas. Lima: Universidad Nacional Agraria La Molina.

LEIGH, E.G.; WINDSOR, D.M. 1982. Forest production and regulation of primary consumers on Barro Colorado Island. In: E.G. Leigh; S. Rand; D.M. Windsor (editors). The ecology of a tropical forest. Seasonal rhythms and long-term changes. Washington: Smithsonian Institution Press. pp. 111-122.

LIETH, H.L.; WHITTAKER, R.H. 1975. Primary productivity of the biosphere. Berlin: Springer-Verlag. 339 pp.

MEDINA, E.; KLINGE, H. 1983. Productivity of tropical forests and tropical woodlands. In: Enc Plant Physiol, 12D: 281-303.

MORROW, P.A. 1984. Assessing the effects of herbivory. In: E. Medina; H.A. Mooney; C. Vásquez-Yanes (editors). Physiological ecology of plants of the wet tropics. The Hague: Dr. W. Junk Publishers. pp. 225-231.

MURPHY, P.G. 1975. Net primary productivity in tropical terrestrial ecosystems. In: H. Lieth; R.H. Whittaker (editors). Primary productivity of the biosphere. Berlin: Springer-Verlag. pp. 217-231.

MÜLLER, D.; NIELSEN, J. 1965. Production brute, pertes par respiration et production nette dans la foret ombrophile tropicales. In: Det forstlige forsgsvaesen, 29: 73-160. 
NEBEL, G.; KVIST, L.P.; VANCLAY, J.K.; CHRISTENSEN, H.; FREITAS, L.; RUÍZ, J. 1999a. Structure and floristic composition of flood plain forests in the Peruvian Amazon: I. Overstorey. Copenhagen: Royal Veterinary and Agricultural University, Department of Economics and Natural Resources, Unit of Forestry. Unpublished manuscript.

NEBEL, G.; DRAGSTED, J.; VANCLAY, J.K. 1999b. Structure and floristic composition of flood plain forests in the Peruvian Amazon: II. The understorey of restinga forests. Copenhagen: Royal Veterinary and Agricultural University, Department of Economics ad Natural Resources, Unit of Forestry. Unpublished manuscript.

NEBEL, G.; KVIST, L.P.; VANCLAY, J.K.; VIDAURRE, H. 1999c. Forest dynamics in flood plain forests in the Peruvian Amazon: Effects of disturbance and implications for management and conservation. Copenhagen: Royal Veterinary and Agricultural University, Department of Economics and Natural Resources, Unit of Forestry. Unpublished manuscript.

PPN DATABASE. 1999. http://daaci.esd.ornl.gov/npp/npp_home.html. March 2. 1999.

PROCTOR, J. 1983. Tropical forest litterfall I. Problems of data comparison. In: S.L. Sutton; T.C. Whitmore; A.C. Chadwick (editors). Tropical rain forest: Ecology and management. Oxford: Blackwell Scientific Publications. pp. 267-273.

1984. Tropical forest litterfall II. The data set. In: A.C. Chadwick; S.L. Sutton (editors). Tropical rainforest: The Leeds symposium. Leeds Philosophical and Literary Society, Leeds. pp. 83-113.

RICHARDS, P.W. 1996. The tropical rain forest. Cambridge: Cambridge University Press. 575 pp.

RYAN, M.G.; HUBBARD, R.M.; CLARK, D.A.; SANFORD, R.L. 1994. Woodytissue respiration for Simarouba amara and Minquartia guianensis, two tropical wet forest trees with different growth habitats. In: Oecología, 100: 213-220. 
SCHLÜTER, U.B.; FUREH, V.B.; JOLY, C.A. 1994. Physiological and anatomical adaptations by young Astrocaryum jauari Mart. (Arecaceae) in periodically inundated biotopes of Central Amazonia. In: Biotropica, 25(4): 384-396.

UNESCO. 1978a. Gross and net primary production and growth parameters. Tropical forest ecosystems. A state-of-knowledge report. Unesco/UNEP/FAO. pp. 233-248.

1978b. Secondary production. Tropical forest ecosystems. A state-ofknowledge report. Unesco/UNEP/FAO. pp. 249-255.

WHITTAKER, R.H.; LIKENS, G.E. 1975. The biosphere and man. In: H. Lieth; R.H. Whittaker (editors). Primary productivity of the biosphere. Berlin: Springer-Verlag. pp. 305-328.

WHITTAKER, R.H.; MARKS, P.L. 1975. Methods of assessing terrestrial productivity. In: H. Lieth; R.H. Whittaker (editors). Primary productivity of the biosphere. Berlin: Springer-Verlag. pp. 55-1 18.

WINT, G.R.W. 1983. Leaf damage in tropical rain forest canopies. In: S.L. Sutton; T.C. Whitmore; A.C. Chadwick (editors). Tropical rain forest: Ecology and management. Oxford: Blackwell Scientific Publications. pp. 229-239.

WORBES, M. 1986. Lebensbedingungen und Holzwachstum in zentralamazonischen Überschwenimungswáldern. In: Scripta Geobotanica, 17: 7-112.

1997. The forest ecosystem of the floodpiains. In: W.J. Junk (editor). The central Amazon floodplain. Ecology of a pulsing system. Berlin: SpringerVerlag. pp. 223-266.

WRIGHT, S.J.; CORNEJO, F.H. 1990. Seasonal drought and the timing of flowering and leaf fall in a Neotropical forest. In: K.S. Bawa; M. Hadley (editors). Reproductive ecology of tropical forest plants. Paris: UNESCO. pp. 49-61. 


\section{Figura 1}
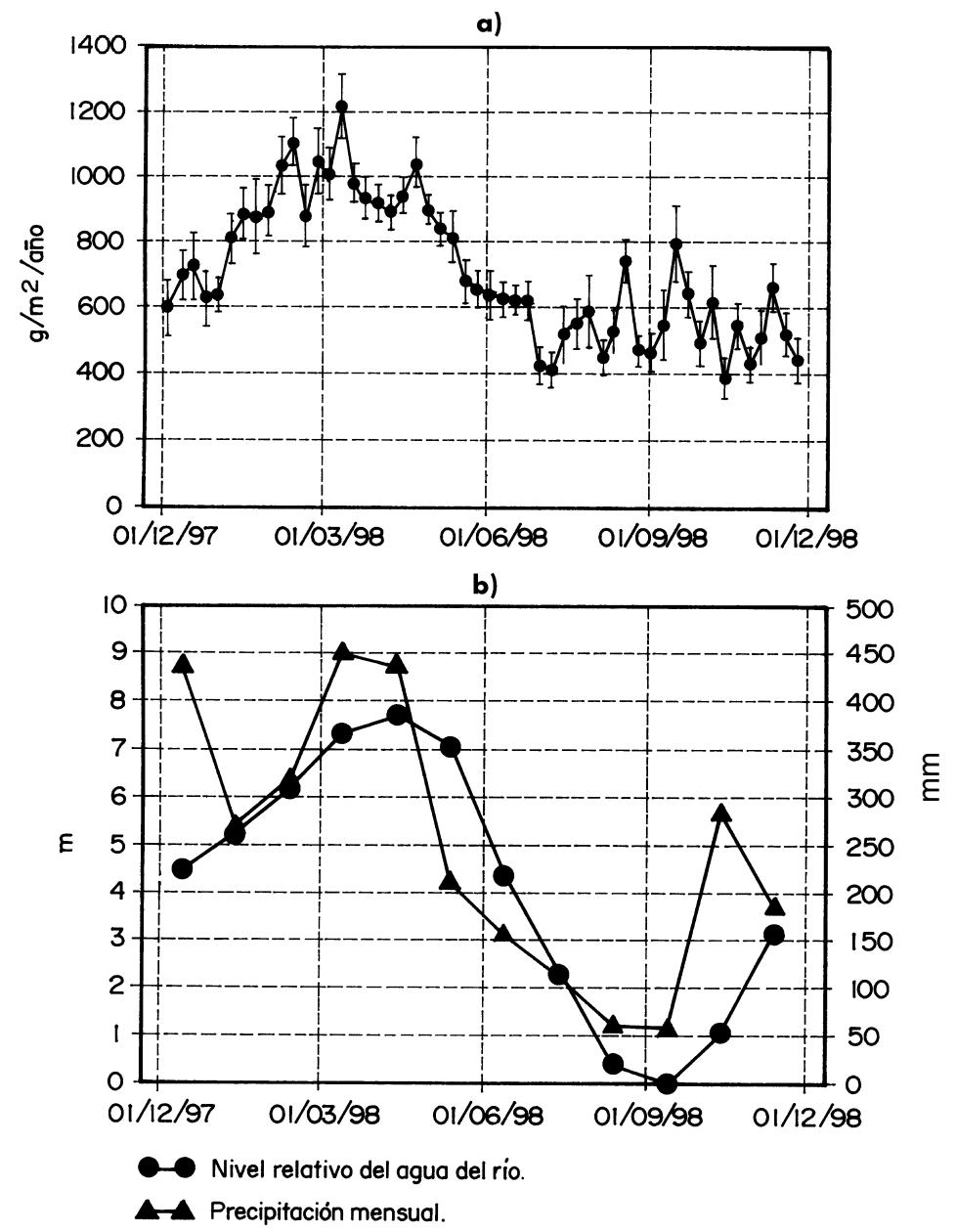

Fig. 1. a) Promedio y límites al $95 \%$ de confianza de 52 colecciones semanales de detritos finos en 75 trampas de detritos colocados en tres bosques de la llanura aluvial inundable. Los valores semanales fueron recalculados para expresar $\mathrm{g} / \mathrm{m}^{2} / a n ̃ o$. b) Eje izquierdo y cruces: promedio relativo mensual del nivel del agua en el río Ucayali en Jenaro Herrera en el período de setiembre 1987 a febrero 1997. Eje derecho y cuadrados: precipitación mensual en Jenaro Herrera en el periodo de diciembre 1997 a diciembre 1998. 


\section{Figura 2}

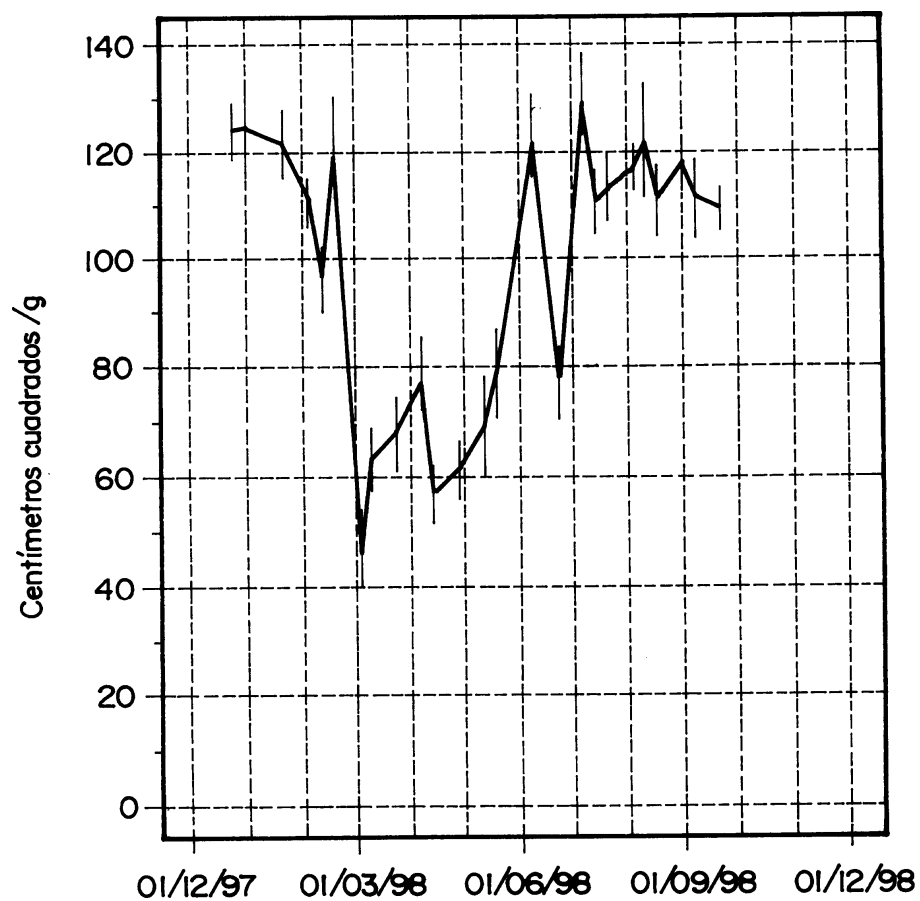

Fig. 2. Promedio específico del área de la hoja y límites al 95\% de confianza para las hojas de 25 colecciones mensuales de detritos. Para calcular las áreas específicas de hoja, se usaron semanalmente todas las hojas de 75 trampas de detritos situadas en tres bosques de la llanura aluvial inundable. 